\title{
Characterizing the surface texture of a dense suspension undergoing dynamic jamming
}

\author{
Olav Rømcke ${ }^{1}$ (D) $\cdot$ Ivo R. Peters ${ }^{2}$ (D) $\cdot$ R. Jason Hearst ${ }^{1}$ (D)
}

Received: 28 June 2021 / Revised: 3 September 2021 / Accepted: 16 September 2021 / Published online: 12 October 2021

(c) The Author(s) 2021

\begin{abstract}
Measurements of the surface velocity and surface texture of a freely propagating shear jamming front in a dense suspension are compared. The velocity fields are captured with particle image velocimetry (PIV), while the surface texture is captured in a separated experiment by observing a direct reflection on the suspension surface with high-speed cameras. A method for quantifying the surface features and their orientation is presented based on the fast Fourier transform of localized windows. The region that exhibits strong surface features corresponds to the the solid-like jammed region identified via the PIV measurements. Moreover, the surface features within the jammed region are predominantly oriented in the same direction as the eigenvectors of the strain tensor. Thus, from images of the free surface, our analysis is able to show that the surface texture contains information on the principle strain directions and the propagation of the jamming front.
\end{abstract}

\section{Graphic Abstract}

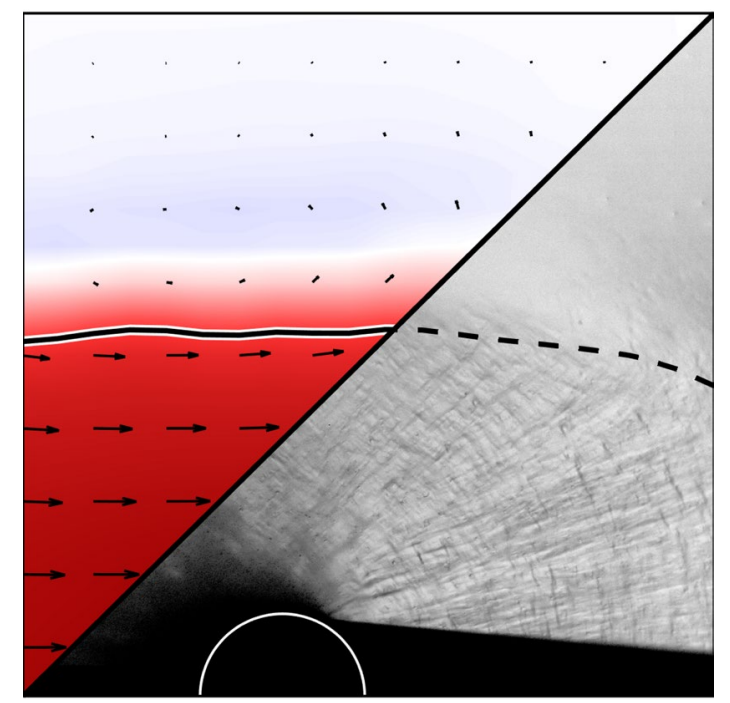

R. Jason Hearst

jason.hearst@ntnu.no

1 Department of Energy and Process Engineering, Norwegian University of Science and Technology, Kolbjørn Hejes vei 2, NO-7491 Trondheim, Norway

2 Faculty of Engineering and Physical Sciences, University of Southampton Highfield, Southampton SO17 1BJ, UK

\section{Introduction}

Suspensions of hard spheres in a Newtonian fluid are known to jam at a critical volume fraction (Krieger 1972). That is, beyond a critical concentration of particles, flow ceases, and a finite yield stress is observed Brown and Jaeger (2014). However, for some suspensions, such as cornstarch and water, a jammed state is accessible for volume fractions lower than the critical volume fraction when stress is applied 
(Wyart and Cates 2014; Peters et al 2016). This rather counter intuitive phenomenon is called dynamic jamming or shear jamming, where the suspension appears fluid-like at low stress, but shear thickens and even jams with sufficiently high stress. As such, a sudden impact causes the suspension to jam (Waitukaitis and Jaeger 2012; Jerome et al 2016), which explains how it is possible to stay afloat while running over a cornstarch suspension (Mukhopadhyay et al 2018; Baumgarten and Kamrin 2019).

The assumption of smooth, force free particles in nonBrownian, non-inertial systems, where viscosity is only a function of the volume fraction, $\phi$ (Stickel and Powell 2005), does not capture this behavior. In real suspensions, particle-particle interactions are an important contributor to the observed behavior (Lin et al 2015; Gadala-Maria and Acrivos 1980; Brown and Jaeger 2014). Between particles, it has been identified that friction (Mari et al 2014; Singh et al 2018; Sivadasan et al 2019; Tapia et al 2019; Madraki et al 2017; Fernandez et al 2013) and repulsive forces (Brown and Jaeger 2014; James et al 2018; Guy et al 2015) are underlying mechanisms for understanding this phenomenon. With a sufficient amount of applied stress, the particles overcome the repulsive force and are brought into frictional contact. At sufficiently high particle concentrations, the contacts form a network capable of supporting the applied stresses.

In sufficiently large domains, the transition from fluid-like to solid-like is observed as a front of high shear rate that propagates from the perturbing body through the suspension and leaves a jammed state in its wake (Waitukaitis and Jaeger 2012; Peters and Jaeger 2014; Han et al 2016; Peters et al 2016; Majumdar et al 2017; Han et al 2018, 2019b; Baumgarten and Kamrin 2019; Rømcke et al 2021). In these works, the jamming front is defined by the velocity contour at half the velocity of the perturbing body, i.e., $0.5 U_{c}$. A normalized front propagation factor is used to quantify how fast the front moves, defined by the relation between the speed of the $0.5 U_{c}$-contour and the perturbing body. The front propagation factor is observed to increase with volume fraction and is independent of perturbing speed for sufficiently high velocities (Han et al 2016; Rømcke et al 2021). This phenomenology is caused by an intrinsic strain (Han et al 2019a; Baumgarten and Kamrin 2019) which is needed in order for the material to build a frictional contact network capable of supporting the applied stresses. The strain level decreases with increasing volume fraction and has an inverse relationship with the front propagation factor (Han et al 2019a).

Most measurement set-ups employed to investigate this problem have a free surface, and as such, the effects of the free surface have been identified as an important question in suspension flow (Denn et al 2018). One interesting feature is the existence of two statically stable states (Cates et al 2005; Cates and Wyart 2014) known as granulation. The material can exist as a flowable droplet with a shiny surface, or in a stressed state as a jammed, pasty granule upheld by capillary forces. A closely linked observable surface feature in dense suspension flow is dilation (Brown and Jaeger 2012; Jerome et al 2016; Maharjan et al 2021). For a sufficiently dense suspension, the granular structure expands under shear, which sets up a suction in the liquid phase. Dilation can thus be observed at the free surface as a transition from reflective to matte as individual particles protrude through the liquid-air interface. Dilation is associated with a large increase in stress (Maharjan et al 2021), and coupled with the suspending fluid pressure (Jerome et al 2016) is able to explain the fluid-solid transition observed in impact experiments with a solid sphere. For a shear jamming front under extension, a reflective-matte transition is observed when the front interacts with the wall (Majumdar et al 2017).

A corrugated free surface has been reported for a wide range of particle sizes and packing fractions and in several experimental setups (Loimer et al 2002; Timberlake and Morris 2005; Singh et al 2006; Kumar et al 2016). In the inclined plane experiment by Timberlake and Morris (2005), two dimensional (2D) power spectra of free surface images indicate that the features exhibit anisotropy, specifically, the corrugations are shorter in the flow direction. Probably more applicable to the work herein is that of Loimer et al (2002) who conducted experiments in an approximately 2D belt driven shear cell with the free surface normal in the vorticity direction. Power spectra in the flow and gradient directions, respectively, also indicate anisotropy. However, how these features appear in the full 2D power spectra remains unclear. The deformation of the free surface is a result of shear induced normal stresses (Timberlake and Morris 2005; Brown and Jaeger 2012), typically observed in dense suspensions (Brown and Jaeger 2014; Guazzelli and Pouliquen 2018; Denn et al 2018). That is, upon shearing, the material responds with a force normal to the confining boundary. Although several experiments investigating the dynamic jamming front phenomenon exhibit a large free surface (Peters and Jaeger 2014; Han et al 2018; Rømcke et al 2021), few studies have dedicated attention to the developing surface texture as the front propagates through the suspension (Allen et al 2018).

In this work, we present observations of the free surface texture as the jamming front propagates unimpeded through the suspension. The aim of the method presented here is to draw quantifiable information from high-speed photographs of the free surface alone, without the need for more complex techniques, e.g., particle image velocimetry (PIV). The result from the free surface images is compared with the velocity field, front propagation and the strain tensor acquired from PIV measurements. 
Fig. 1 Schematic of the experimental set-up. a PIV set-up with two cameras, $\mathbf{b}$ texture set-up with the backlit acrylic plate and $\mathbf{c}$ birdseye-view of the suspension surface with the dotted square indicating the investigation region of the present study. In the images, the beige layer represents the suspension, and the blue layer beneath it represents a layer of Fluorinert

\section{Experimental procedure}

The data used here are collected from two different experiments. A single cylinder is traversed through a layer of cornstarch and sucrose-water suspension. First, as a reference, the free surface was seeded with black pepper. High-speed images of the suspension surface were captured under indirect lighting. PIV was conducted on these particle images, resulting in a time resolved velocity field. Secondly, by minor adjustments to the set-up, we record the free surface. In this case, the suspension is not seeded, while the camera was positioned such that it observed a direct reflection on the free surface, enhancing the visibility of any surface features.

The experimental set-ups are shown in Fig. 1. Both experiments are conducted in a $1 \mathrm{~m} \times 0.5 \mathrm{~m}$ tank. The tank is first filled with a $15-\mathrm{mm}$-layer of high density, low viscosity Fluorinert oil (FC74) (Loimer et al 2002; Peters and Jaeger 2014; Han et al 2018; Rømcke et al 2021), followed by a 15-mm-thick suspension layer consisting of cornstarch (Maizena maisstivelse) and a sucrose-water solution (50\% wt) at a nominal volume fraction of $\phi=0.36$ (Rømcke et al 2021), defined as

$\phi=\frac{(1-\beta) m_{s} / \rho_{s}}{(1-\beta) m_{s} / \rho_{s}+m_{l} / \rho_{l}+\beta m_{s} / \rho_{w}}$.

Here, $\beta=11 \%$ is the water content in the starch, while $m_{s}$ and $m_{l}$ are the measured mass of starch and sucrose solution, respectively. The densities of the starch, sucrose solution and water are $\rho_{s}=1.63 \mathrm{~g} / \mathrm{ml}, \rho_{l}=1.23 \mathrm{~g} / \mathrm{ml}$ and $\rho_{w}=1.0 \mathrm{~g} / \mathrm{ml}$, respectively. We mix the suspension for two hours before it is loaded into the tank. The suspension floats atop the denser Fluorinert $(\sim 1.8 \mathrm{~g} / \mathrm{ml})$, which ensures a near stress free bottom boundary and makes the system approximately $2 \mathrm{D}$ (Peters and Jaeger 2014). A 25-mm-diameter $(D)$ cylinder is submerged in the suspension and is traversed at a velocity of $U_{c}=0.14 \mathrm{~m} / \mathrm{s}$; the effect of changing $U_{c}$ is the subject of a previous study (Rømcke et al 2021). Both the cylinder velocity $\left(U_{c}>0.06 \mathrm{~m} / \mathrm{s}\right)$ and volume fraction $\left(\phi_{m}<\phi<\phi_{0}\right)$ are in a range where dynamic jamming is known to occur for this suspension (Rømcke et al 2021).

The suspension is pre-sheared by towing the cylinder back and forth equivalent to an actual run, before any measurements are taken. When capturing particle images for the PIV, two 4 megapixel high-speed cameras (Photron FASTCAM Mini WX100) view the suspension surface in front

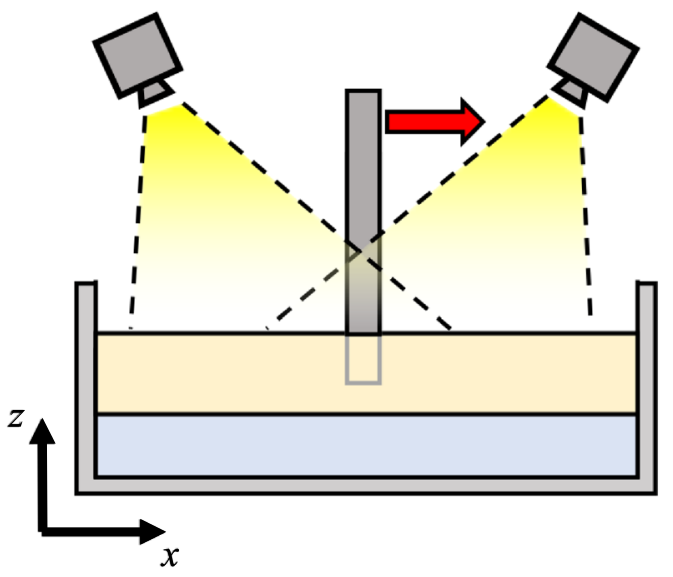

(a)

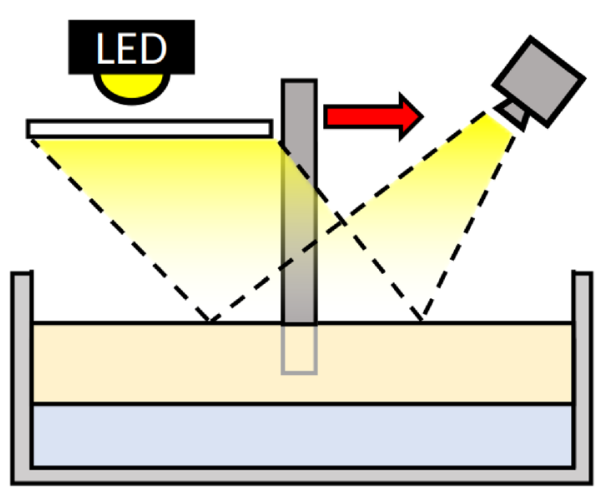

(b)

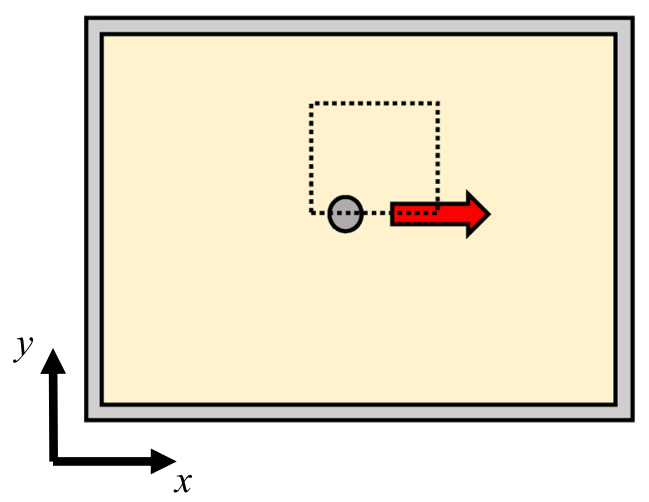

(c) 
and behind the traversing cylinders (Fig. 1a). Pulsed LED lighting was used to illuminate the surface and was synchronized with the camera acquisition at $750 \mathrm{~Hz}$. The particle images were converted to velocity fields with LaVision DaVis 8.4.0 PIV software. An initial pass was performed with 96 pixels $\times 96$ pixels square interrogation windows, followed by two passes with circular interrogation windows with decreasing size ending at 48 pixels $\times 48$ pixels. For all passes, the interrogation windows have a $50 \%$ overlap. The resulting instantaneous velocity fields are stitched together in post processing, masking out the cylinder in each frame. This results in a velocity field fully surrounding the cylinder.

As mentioned above, only minor adjustments to the setup are needed in order to observe the surface features. This is illustrated in Fig. 1b. Here, a single camera is positioned such that it views a direct reflection of a backlit, semitransparent, acrylic sheet on the suspension surface. This enhances any surface features not captured by the PIV; note that the tracer particles used for PIV also interfere with the detection of the surface topology, which is why a separate campaign was used for surface texture measurements. Figure 1c gives a birds eye view of the suspension surface. For the scope of this work, we focus on the region indicated by the dotted square. See Rømcke et al (2021) for details on the full velocity field.

For the texture images, LaVision Davis 8.4.0 was used to find a third order calibration polynomial, mapping the image coordinates $(i, j)$ to the lab coordinates $(x, y)$. MATLAB was used for all further processing of the texture images. Here, for consistency, the results are always plotted in the calibrated lab coordinate system $(x, y)$. However, calculations on the surface features are done in pixel coordinates $(i, j)$, and the results in $(i, j)$ are mapped to $(x, y)$ with the calibration polynomial.

Examples of both the PIV and texture images are shown in Fig. 2. The cylinder position is denoted $x_{c}$, which starts as $x=0$ and moves in the positive $x$-direction. The raw particle images (Fig. 2a-d) do not provide any information on the suspension texture. The resulting velocity fields from the PIV analysis of these images are shown in Fig. 2e-h. Note the sharp transition in velocity that propagates away from the cylinder as it moves through the flow. By the end of an experimental run, the whole field of view is moving with the cylinder as shown in Fig. $2 \mathrm{~h}$. The $0.5 U_{c}$ contour is represented by the black line and used as a proxy for the position of the jamming front as is common in previous studies (Waitukaitis and Jaeger 2012; Peters and Jaeger 2014; Peters et al 2016; Han et al 2016, 2018, 2019b, a; Rømcke et al 2021).
From the velocity data, we estimate the local accumulated strain. The strain is shown to be an important parameter with regards to jamming. Given that the suspension is subjected to a sufficient amount of stress, an intrinsic onset strain dictates the amount of strain needed before the suspension transitions into a jammed state (Majumdar et al 2017; Han et al 2016, 2019a; Rømcke et al 2021). The nominal value of the onset strain depends on the volume fraction (Han et al 2016, 2018, 2019a). We define the strain in the same manner as Rømcke et al (2021). In short, by estimating the movement of the material points $\mathbf{x}_{\mathbf{p}}(\mathbf{X}, t)=\mathbf{X}+\int_{0}^{t} \mathbf{u}\left(\mathbf{x}_{\mathbf{p}}(\tau), \tau\right) d \tau$, we calculate the deformation gradient tensor $\mathbf{F}=\frac{\mathbf{x}_{\mathbf{p}}}{\partial \mathbf{X}}$, where $\mathbf{X}$ is the position of the material points at $t=0$. From the deformation gradient, the left stretch tensor $\mathbf{V}$ is acquired from a polar decomposition $\mathbf{F}=\mathbf{V R}$. The tensor $\mathbf{V}$ has eigenvalues and eigenvectors, $\lambda$ and $\mathbf{n}$, respectively. Here, we employ the Eulerian logarithmic strain tensor (Nemat-Nasser 2004)

$\mathbf{e}=\sum_{i} \ln \left(\lambda_{i}\right) \mathbf{n}_{i} \otimes \mathbf{n}_{i}$.

Eigenvalues are ordered $\lambda_{1}>\lambda_{2}$, such that $\mathbf{n}_{1}$ and $\mathbf{n}_{2}$ signify the direction of stretch and compression, respectively; $\mathbf{n}_{1}$ and $\mathbf{n}_{2}$ are orthogonal. Figure $2 \mathbf{i}-1$ shows the evolution of the norm of the strain tensor $\epsilon=\|\mathbf{e}\|$. The strain $(\epsilon)$ at the jamming front is relatively constant throughout an experiment (Rømcke et al 2021) and measured to be $\sim 0.14$ in the case presented here. In the current work, we will focus on the orientation of the eigenvectors. Though the strain field is relatively homogeneous by the end of an experimental run (Fig. 21), the superimposed eigenvectors show that the direction in which the suspension is stretched and compressed depends on the location in the flow.

Finally, a time series of the texture experiment is presented in Fig. $2 \mathrm{~m}-$ p. Due to the specific lighting conditions explained above, this experiment reveals features not visible in the PIV particle images. Two key observations form the scope of this work. First, like the velocity field, there is a sharp transition between the regions with and without surface structures. This transition propagates from the cylinder, into the suspension, leaving a textured surface in its wake. We observe a change from a reflective to a matte surface indicative of a dilated suspension (Brown and Jaeger 2012; Maharjan et al 2021; Bischoff White et al 2010; Smith et al 2010). Second, by comparing the eigenvectors of the strain tensor (Fig. 2i-l) and the orientation of the surface features (Fig. $2 \mathrm{~m}-\mathrm{p}$ ), there appears to be a connection. More specifically, the eigenvectors and surface features appear to be oriented in the same direction at the same locations in the flow, suggesting the raw surface images may hold quantifiable information akin to the PIV. This is explored further below. 

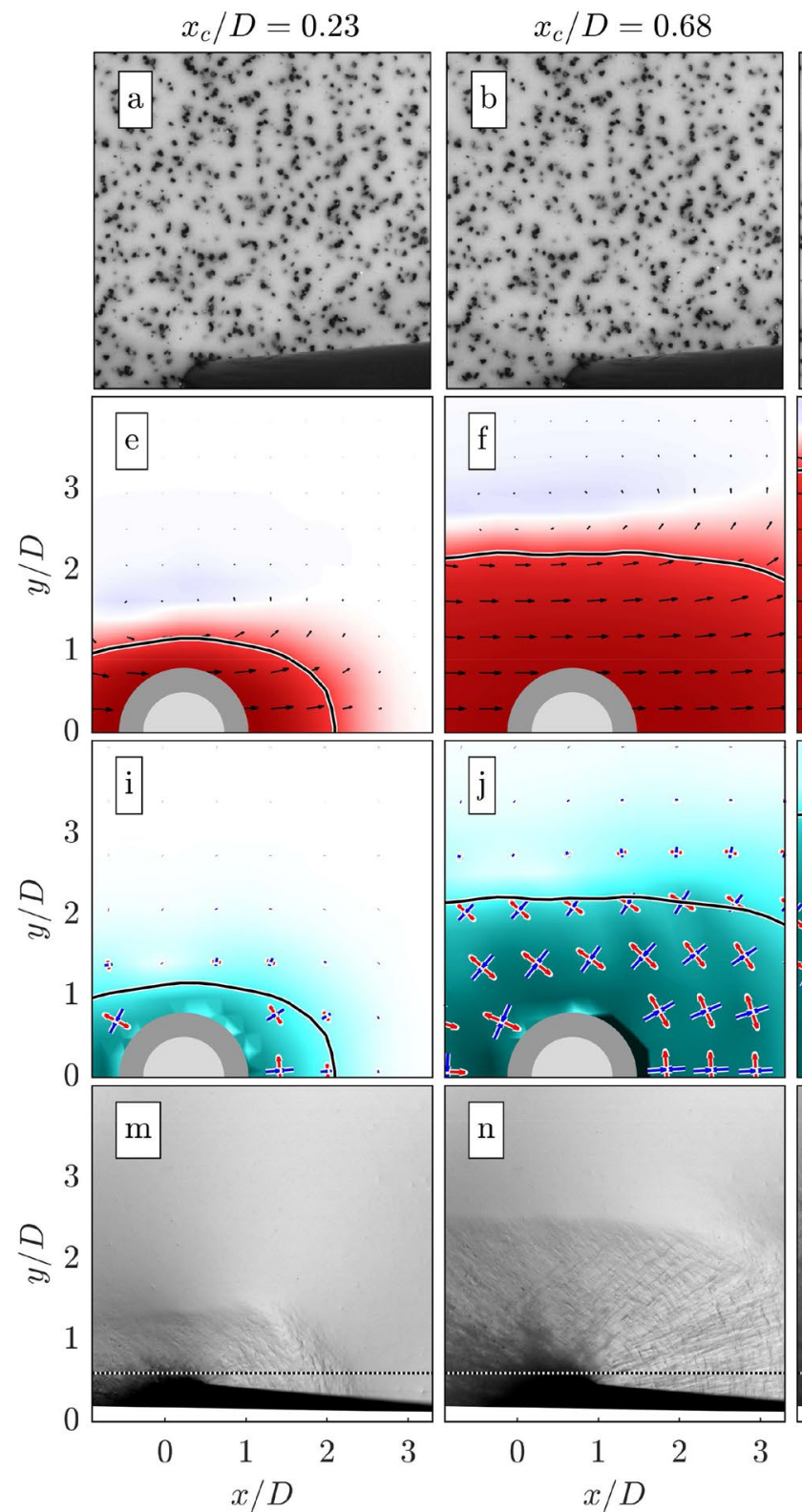

$x / D$

Fig. 2 Time series comparison between PIV and texture images. $x_{c}$ represents the location of the cylinder. a-d Raw PIV particle images. e-h Resulting PIV velocity fields with superimposed velocity vectors. i-l Accumulated strain $(\epsilon)$ with superimposed eigenvectors. Red represents the direction of stretch $\left(\mathbf{n}_{1}\right)$, while blue indicates the direc-

\section{Analysis methodology}

The aim of this study is to quantify the structures observed at the free surface of a dense suspension undergoing dynamic jamming. In this section, we present a method that is able to identify surface features and their orientation. In short, images of the free surface are divided into interrogation windows, and the 2D fast Fourier transform (FFT) of the local windows is used as a basis for quantifying these structures,

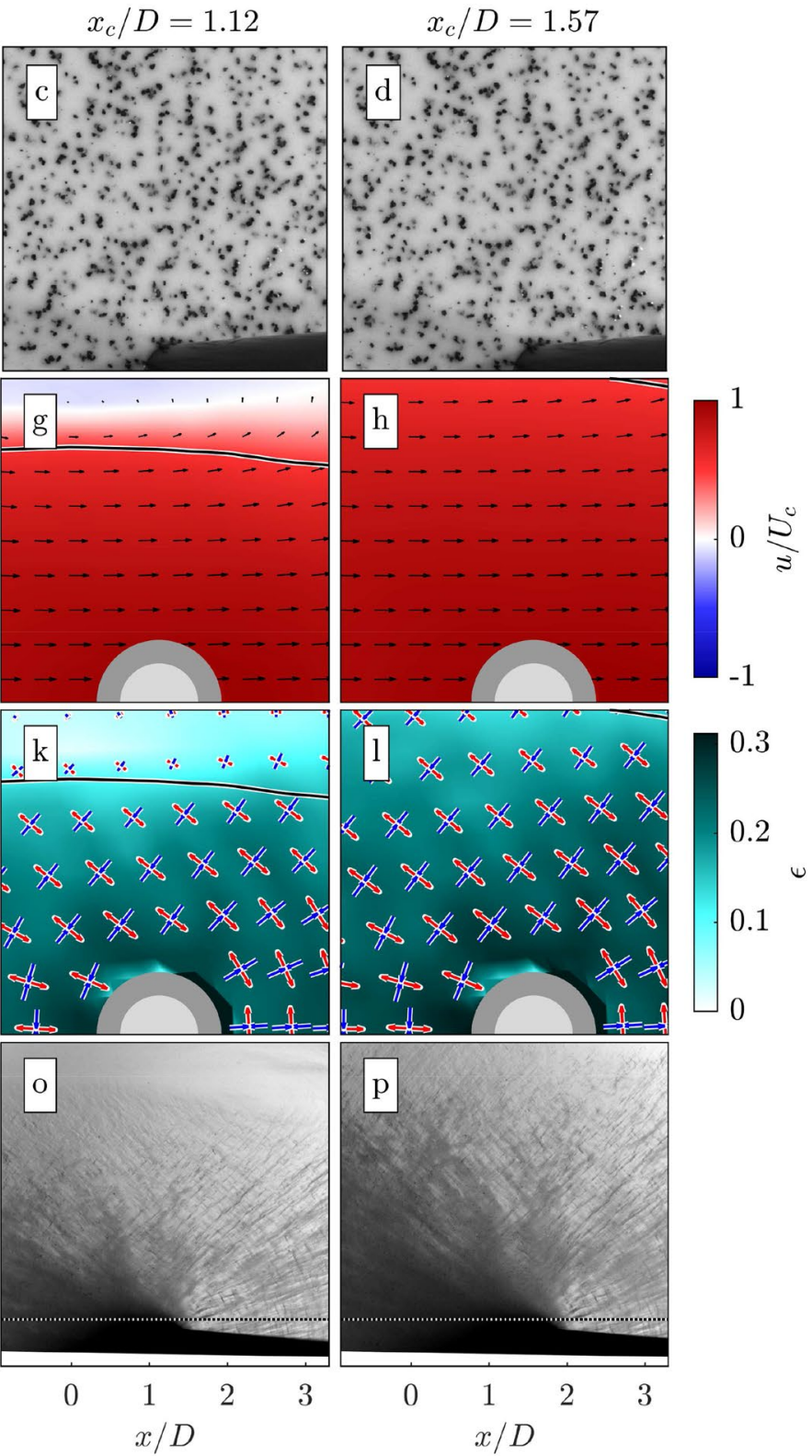

tion of compression $\left(\mathbf{n}_{2}\right)$. For clarity, $\mathbf{n}_{\mathbf{i}}$ are scaled with $\epsilon$. In $\mathbf{e}-\mathbf{l}$, the jamming front $\left(0.5 U_{c}\right.$ contour $)$ is represented by the black line. $\mathbf{m}-\mathbf{p}$ Surface features as the front propagates. In order to avoid the cylinder shadow, the analysis is restricted to the region above the dotted line

which is presented in Sect. 3.1. Section 3.2 establishes a basis by which this process can be optimized and determines the optimal parameter values used in the remainder of this work.

\subsection{FFT and sector averaging}

Here, in order to extract local information from the texture images, such as how dominant the features are and what 

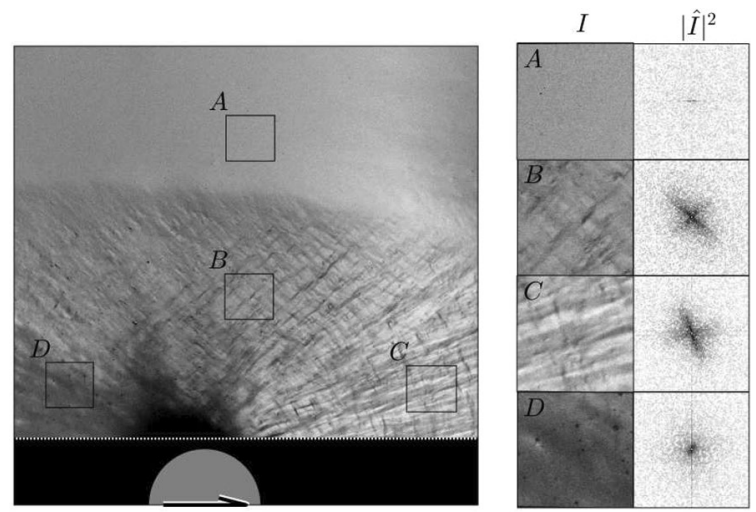

Fig. 3 A snapshot of the suspension surface. Example interrogation windows are presented in the right columns with both the pixel intensity $(I)$ and the 2D power spectra $\left(|\hat{I}|^{2}\right)$. The intensity scale of the power spectra is plotted logarithmically. $200 \times 200$ pixel interrogation windows representing $\mathrm{A}$ no features, $\mathrm{B}$ and $\mathrm{C}$ distinct features, D weak features

orientation they have, we divide the frame into interrogation windows. Figure 3 gives examples of four representative windows, which we will focus on in this section. As a first step toward quantifying the surface features, we take a

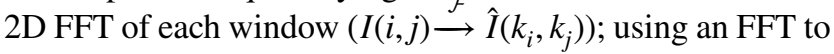
gain information on the surface texture is a common practice for interfacial flows, e.g., (Zhang 1996; Loimer et al 2002; Timberlake and Morris 2005; Singh et al 2006). The mean pixel intensity of the interrogation window is subtracted before calculating the FFT. Here, $I$ and $\hat{I}$ represent the intensity in the image and wave number domain, respectively.

The resulting FFTs of the interrogation windows are seen in the right column of Fig. 3 represented here by the power spectral density $|\hat{I}|^{2}$. Notice how the streaks in the interrogation windows are reflected in the corresponding power spectra. For B and C, the power spectra show clear features orthogonal to the streaks observed in the image. This trend is also observed for D, though clustered at lower wave numbers. Window A, however, has an almost perfectly homogeneous intensity, which is reflected in the power spectrum by predominantly exhibiting values at the noise level. An unwanted feature is also revealed by the FFT. As seen in the power spectra of A and D, a signal is observed along the lines $k_{i}=0$ and $k_{j}=0$. Though the background subtraction removes most of these features, they are still significant for low signal-to-noise ratio regions, e.g., A and D. For any further processing, this issue is addressed by masking out the values at the lines $k_{i}=0$ and $k_{j}=0$.

The general shape of the power spectra encodes both how dominant the features are and in what direction they are oriented. We extract the shape of a power spectrum by taking a sectional average. First, we denote the wave vector $\mathbf{k}=\left(k_{i}, k_{j}\right)$, so that in polar coordinates, $k=|\mathbf{k}|$ with the angle $\theta=\tan ^{-1}\left(k_{j} / k_{i}\right)$. The averaging procedure is taken over the wave number $k$ ranging from 0 to 0.5 pixel $^{-1}$ in sectors of size $\Delta \theta$. In polar coordinates, the average over a sector is represented by the integral

$f(\theta, \Delta \theta)=\frac{8}{\Delta \theta} \int_{0}^{1 / 2} \int_{\theta-\Delta \theta / 2}^{\theta+\Delta \theta / 2}|\hat{I}|^{2}(k, \theta) k \mathrm{~d} \theta \mathrm{d} k$.

Numerically, given the discrete values of $|\hat{I}|^{2}$, the circle is divided into a number $(N)$ of equally spaced angular sectors with a relative overlap $\xi$, shown schematically in Fig. 4a. Note that $\Delta \theta=2 \pi /(N(1-\xi))$. The sector average is calculated as the mean of $|\hat{I}|^{2}$ contained within the sector. Figure $4 \mathrm{~b}$ and $\mathrm{c}$ show how the trend in the power spectra is reflected in the resulting curve $f$.

We use the sector average curve, $f$, to extract key features of a power spectrum. First, we calculate the shape factor, $S$, of the sector average curve in order to distinguish between an interrogation window with and without surface structure. $S$ is a measure of how much a shape resembles a circle. The shape factor is defined such that it compares the perimeter $(\delta)$ of $f$ with the circumference of a circle with the same area $(\alpha)$ as $f$, i.e.,

$S=\frac{2 \sqrt{\pi} \sqrt{\alpha}}{\delta}$.

The shape factor $S$ is in the range 0 to 1 , where 1 represents a perfect circle. The power spectrum of window A from Fig. 3 is relatively uniform and is expected to show values of $S$ close to unity. This can also be seen by substituting in a constant value for $|\hat{I}|^{2}$ in (4) resulting in $f(\theta)=$ constant. Window $\mathrm{C}$, on the other hand, is expected to show values distinguishable from a perfect circle as we observe clear angular dependencies. Secondly, we take the orientation of the peak of $f$ to represent the orientation of the dominant surface features. In the image coordinates $(i, j)$, we denote the orientation of the peak $\theta_{P}^{*}$ which defines the unit

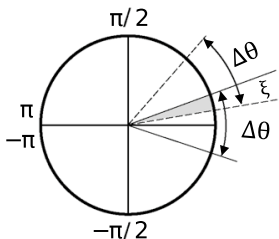

(a)

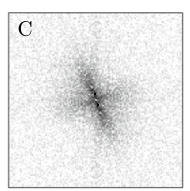

(b)

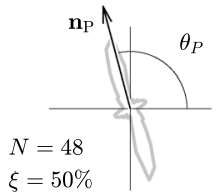

(c)
Fig. 4 Schematics of the sector averaging. a Two neighbouring sectors with overlap. b Power spectra of interrogation window $\mathrm{C}$ with $\mathbf{c}$ resulting sector average curve $(f)$ calculated with $N=48$ sectors and $\xi=50 \%$ overlap. The vector $\mathbf{n}_{P}$ and the angle $\theta_{P}$ represent the orientation of the peak 
vector $\mathbf{n}_{P}^{*}=\left(\cos \theta_{P}^{*}, \sin \theta_{P}^{*}\right)$. The unit vector is mapped to the spatial coordinate system with the calibration, such that $\mathbf{n}_{P}=\left(\cos \left(\theta_{P}\right), \sin \left(\theta_{P}\right)\right)$, where $\theta_{P}$ represents the orientation of the peak in the $(x, y)$-system. Note that the Fourier transform is symmetric about the origin, thus two equal sized peaks separated by an angle $\pi$ are observed in Fig. 4c. As a representation of the main direction of the structures, we only focus on peaks in the upper half plane $0 \leq \theta_{P}<\pi$.

\subsection{Determining processing parameters}

Some parameters have been presented in the previous section that affect the sector average curve $f$. Here, we focus on the size of the interrogation window, as well as the number of sectors $(N)$ and the sector overlap $(\xi)$. Adjusting these parameters will consequently have an impact on $S$ and $\theta_{P}$. As one of our aims is to be able to distinguish between regions with surface features (such as window C) from regions without surface features (such as window A), we choose to use a set of processing parameters that will maximize the difference in $S$ between these two scenarios.

Trends of $S$ for window A and C over a range of processing parameters $(N$ and $\xi)$ are presented in Fig. 5. As expected, the sector average curve, $f$, for window A (Fig. 5a) takes on a circular shape, while for window $\mathrm{C}$ (Fig. 5b), the curve indicates clear peaks orthogonal to the streaks on the surface. This is also reflected by the values of $S$ showing higher values for window A compared to C.

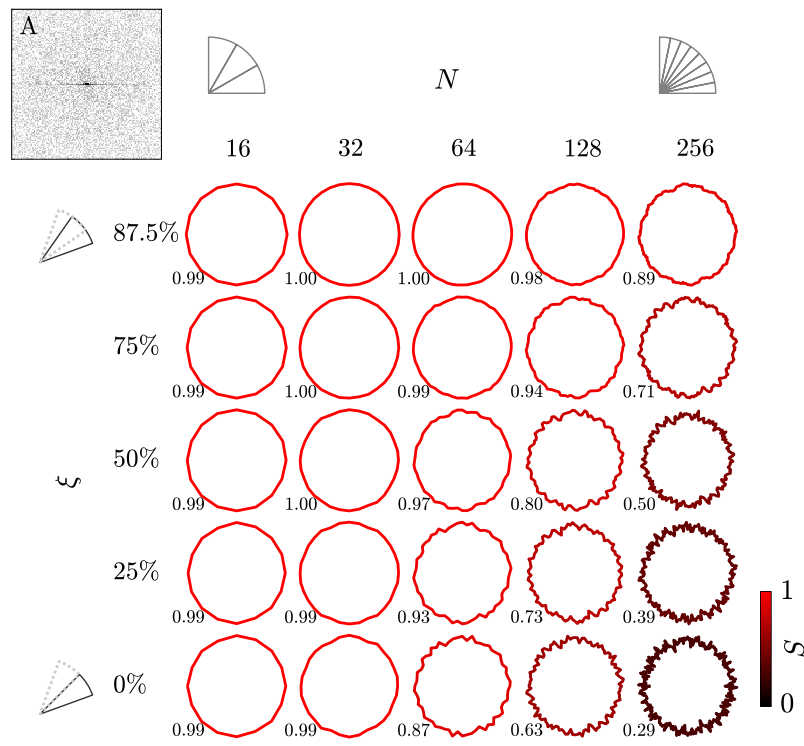

(a)

Fig. 5 Sector average curves $(f)$ for $256 \times 256$ pixels interrogation windows of $\mathbf{a}$ A and $\mathbf{b} \mathbf{C}$ over a range of overlap $(\xi)$ and number of sectors $(N)$. The resulting shape factor $(S)$ is indicated by the
However, in the extreme cases, the difference in $S$ tends to be small. For example, choosing a large number of sectors and a small overlap, even though the the resulting sector average curve from $\mathrm{C}$ shows clear peaks and a low shape factor, the shape factor of $\mathrm{A}$ is also reduced. On the other hand, choosing few sectors with a large overlap yields an almost perfect circular result for window $\mathrm{A}$, but the peaks in $\mathrm{C}$ are no longer clear and the shape factor is higher. Figure 5 indicates that there is an optimal combination of $N$ and $\xi$ that would maximize the difference in shape factor $S$, such that the shape factor yields a clear distinction between the two scenarios.

We point out that the result presented in Fig. 5 only compares two locations in the flow (A and $\mathrm{C}$ ) for one window size $(256 \times 256$ pixels $)$ from a specific snapshot of the flow. However, the trend is the same when comparing window $A$ with window $B$ and $D$ over a range of window sizes from $64 \times 64$ to $512 \times 512$ pixels. After considering different window sizes and values for $N$ and $\xi$, the overall difference in $S$ appears to converge with increasing window size at $256 \times 256$ pixels. Setting $N=128$ and $\xi=75 \%$ gives a sufficiently large difference in $S$, while simultaneously ensuring a satisfactory angular resolution for establishing $\theta_{P}$. For the remainder of this work, we will use a window size of $256 \times 256$ pixels, with $N=128$ and $\xi=75 \%$ when computing the sector average curve, $f$. It would be important to note that the "optimal" is dependent on the specific experimental set-up and that the results found here would

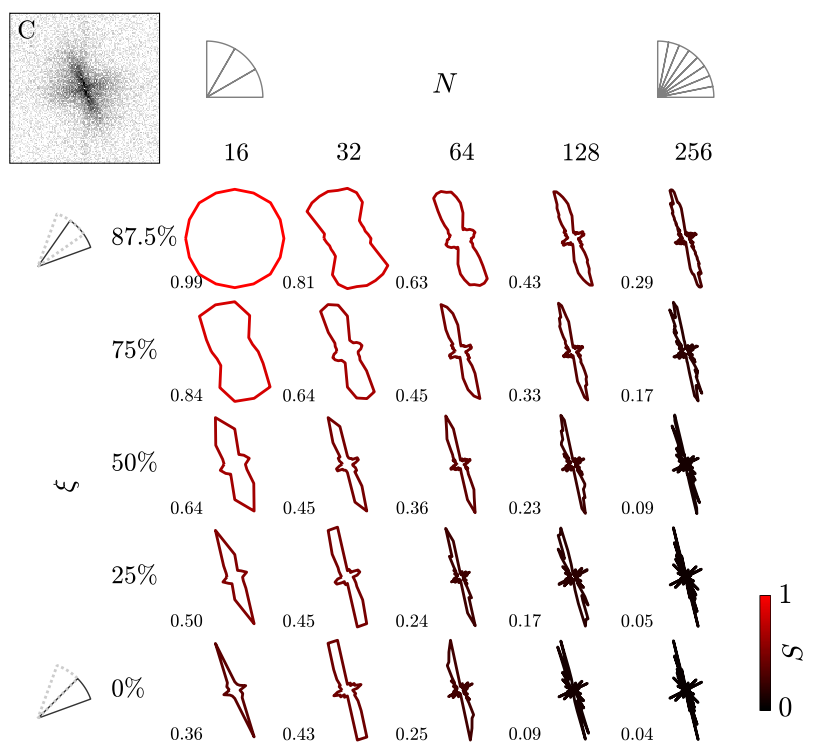

(b)

color scheme and also presented numerically for each curve. For the remainder of this work, we choose $N=128$ and $\xi=75 \%$ 
not be universally optimal akin to how PIV processing parameters are optimized individually for each experiment.

\section{Results}

The method outlined in Sect. 3 is now applied to the full texture images. Section 4.1 presents results from the texture images alone. By setting a threshold value for $S$, we identify the textured region, and in Sect. 4.2, we give an estimate of how fast this region propagates into the suspension. In Sect. 4.3, the data extracted from analysing the texture images are directly compared to the PIV data. First, we present the combined evolution of the shape factor and velocity field. We then show that in certain regions of the flow, the direction of the texture and the eigenvectors of the strain tensor are predominantly oriented in the same direction.

\subsection{Quantifying the texture for the full field}

From the method presented above, we are capable of identifying if the surface shows features $(S)$, and in what direction the features are oriented $\left(\mathbf{n}_{P}\right)$. Both $S$ and $\mathbf{n}_{P}$, are presented in Fig. 6. The analysis is conducted on the full field of the time series shown in Fig. $2 m-$ p, with the processing parameters found in Sect. 3.2. In addition, we let neighbouring interrogation windows overlap with $75 \%$.

Figure 6a shows that the shape factor clearly separates the flow into two regions. Five iso-contours, $S=0.6,0.7$, $0.8,0.85$ and $S=0.9$, are superimposed on the $S$-field. Here, these contours tend to cluster at the transition between the textured and texture-free surface. Analogous to the $0.5 U_{c}$ contour being used to identify the position of the jamming front from PIV data, a contour level between $S=0.6$ and $S=0.9$ identifies the position of the front from the texture data. In the later stages of an experimental run, dilation renders the surface matte. Due to the increase in $S$ observed
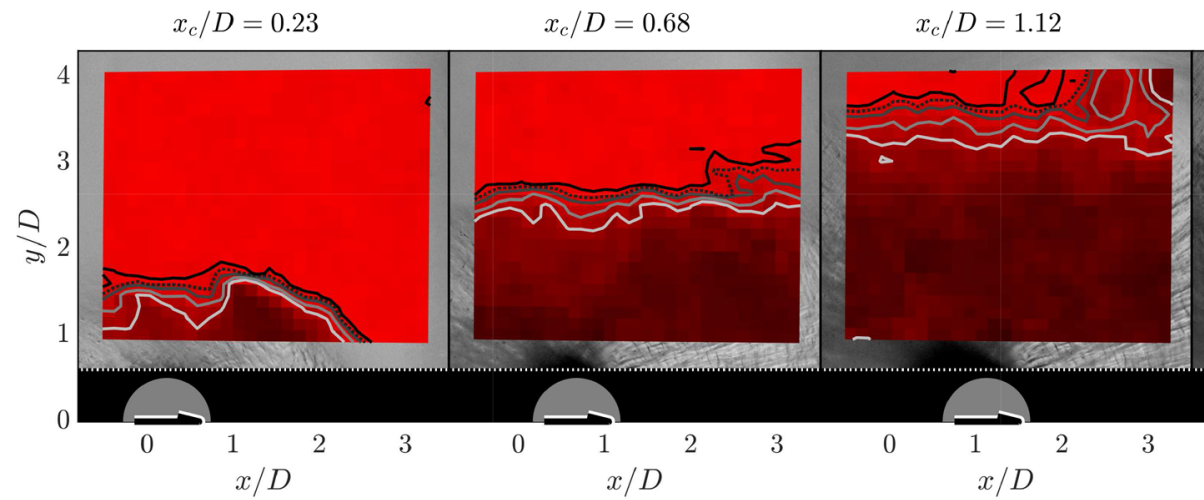

$x_{c} / D=1.57$

(a)

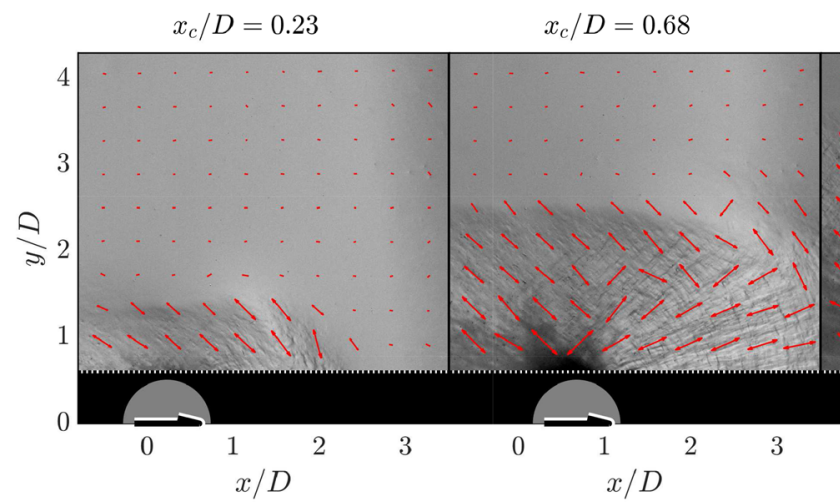

$x_{c} / D=1.12$

$x_{c} / D=1.57$

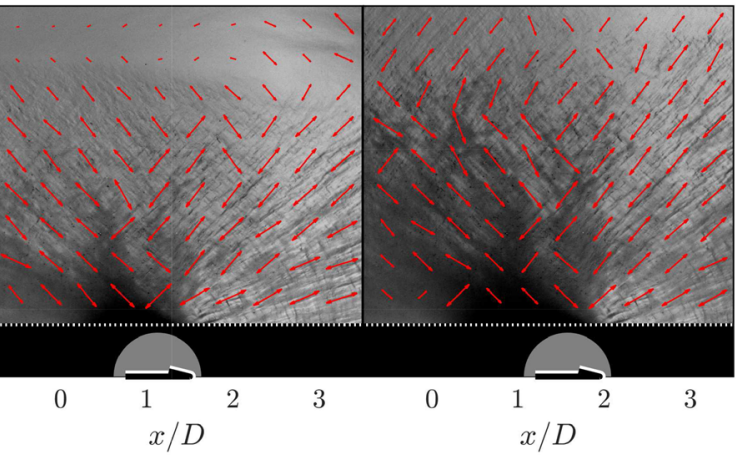

(b)

Fig. 6 Texture analysis on the full field of the snapshots in Fig. $2 \mathrm{~m}-\mathrm{p}$. a Shape factor $S$. The contours represent $S=0.6,0.7,0.80 .85$ and 0.9 , where the 0.85 is represented by the dotted line. b Orientation of the peak $\left(\mathbf{n}_{P}\right)$ from the sector average, which is represented by the vectors superimposed on the corresponding surface image. Every third vector is plotted to reduce clutter, and scaled with $1-S$. In addition, $\mathbf{n}_{P}$ is rotated by $\pi / 2$ such that the vectors point in the same direction as the surface streaks rather than normal to them 
in Fig. 6a, it becomes increasingly difficult to identify the preferred texture orientation in some parts of the flow. This is most noticeable in the wake of the cylinder.

The corresponding orientation of the surface features are indicated in Fig. 6b. As noted in Figs. 4 and 5, the peak in $f$ is oriented normal to the direction of the dominant streaks in the texture image. For clarity in Fig. 6b, the vectors indicating the peak angle, $\mathbf{n}_{P}$, are rotated by $\pi / 2$ such that they are oriented parallel to the surface streaks. Here, we plot both $\mathbf{n}_{P}$ and $-\mathbf{n}_{P}$, representing the symmetry of the power spectra.

The orientation of the vectors can be directly compared to the actual texture image. Notice that fore (aft) of the cylinder, the vectors generally tend to be forward (backward) leaning, reflecting the dominant streaks in the region. The region roughly around the same $x$-location as the cylinder, exhibits a crosshatch pattern (Chang et al 1990; Albrecht et al 1995). This is more clearly indicated in Fig. 3 by window B. As we only report the most dominant peak in $f$, the vectors $\mathbf{n}_{P}$ generally show a mix of forward-leaning and backward-leaning in this region. Notice the similarity with the eigenvectors plotted in Fig. $2 \mathbf{i}-1 ; \mathbf{n}_{1}$, representing direction of stretch, is backwards-leaning, while $\mathbf{n}_{2}$, representing compression, forward leaning. The similarity between $\mathbf{n}_{P}$ and the strain eigenvectors will be addressed in greater detail in Sect. 4.3.

\subsection{Propagation of the texture transition}

Figure 7 establishes the location of the texture transition and estimates its propagation velocity into the suspension. Here, we focus on the transverse direction relative to the cylinder velocity. As with the jamming front position being identified by the $0.5 U_{c}$ contour, we will identify the texture transition by a contour value in $S$. We will compare how fast the jamming front and texture transition propagates into the suspension.

The front propagation factor, denoted here by $\kappa$, is defined as the relation between the speed of the jamming front and the speed of the perturbing body. In the transverse direction relative to the cylinder velocity, the front propagation factor is the time derivative of the jamming front's $y$-position $\left(y_{f}\right)$ relative to the speed of the cylinder $\left(U_{c}\right)$. Since

$\kappa=\frac{\mathrm{d} y_{f} / \mathrm{d} t}{U_{c}} \approx \frac{\Delta y_{f} / \Delta t}{\Delta x_{c} / \Delta t}=\frac{\Delta y_{f}}{\Delta x_{c}}$,

rather than estimating a time derivative, we will focus on the equivalent relation $\Delta y_{f} / \Delta x_{c}$. As such, for the texture data, we start by establishing the position of the texture transition.

As seen by the superimposed contours in Fig. 6a, the position of the texture transition depends on the choice of contour level. Figure 7 a shows vertical cross sections of the shape factor taken as the cylinder translate in the $x$-direction

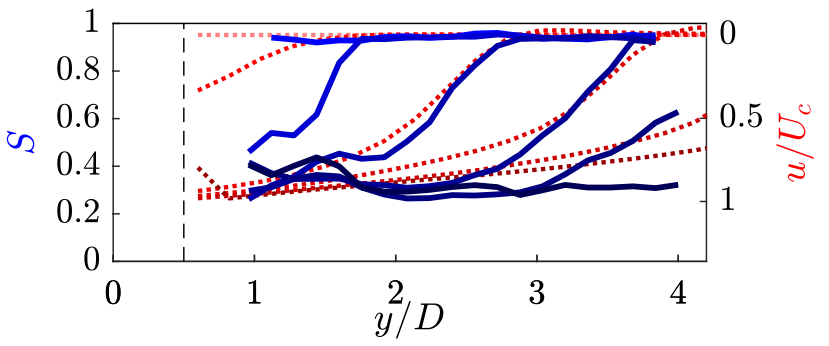

(a)

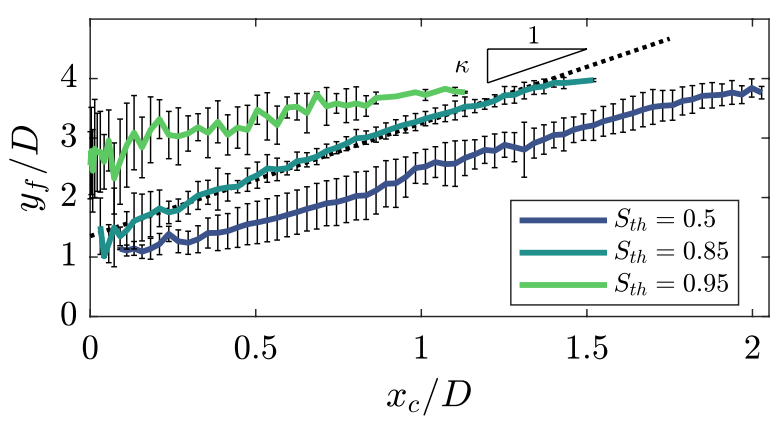

(b)

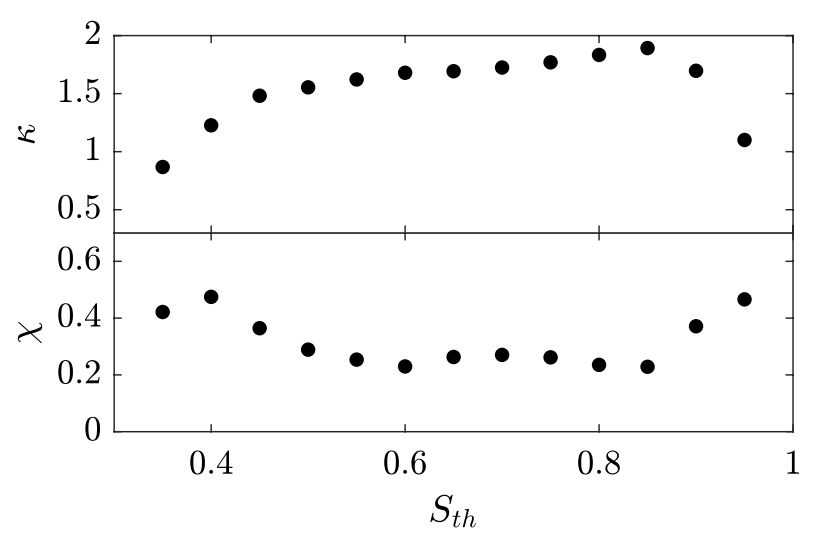

(c)

Fig. 7 a Profiles of the shape factor (solid blue lines) and velocity (dotted red lines) for an experimental run in vertical cross sections taken at the location of the cylinder $\left(x=x_{c}\right)$. The black dashed line indicates the location of the cylinder surface. The color intensity indicates different $x_{c}$ as the cylinder moves through the flow. Velocity profiles are flipped to emphasize the similarity between $S$ and $u$ b Position of the front, $y_{f}$, for three different threshold values of the shape factor. The slope of the linear regression line is denoted $\kappa$. c Slope, $\kappa$, and RMS of the error of the regression line, $\chi$, as functions of the threshold value, $S_{t h}$

$\left(S\left(x=x_{c}, y\right)\right.$, where $x_{c}$ represents the instantaneous cylinder position). As with the velocity profiles (indicated by the dotted lines in Fig. 7a), $S$ is not a perfect step, and as pointed out above, the position of the front will depend on the choice of the contour level. We use $y_{f}$ to denote the position of the texture transition and is given implicitly from the shape factor profiles as $S_{t h}=S\left(x=x_{c}, y_{f}\right)$. Here, $S_{t h}$ represents the 
contour level, or the threshold value for $S$ separating textured from texture free surface. Numerically, the front position is acquired by linearly interpolating the shape factor profiles, e.g., Fig. 7a.

The resulting front position $\left(y_{f}\right)$ from all experimental runs is plotted in Fig. $7 \mathrm{~b}$ for three different values of $S_{t h}$. Due to variation in the front position shown in Fig. 7b, calculating the relation $\Delta y_{f} / \Delta x_{c}$ directly has large uncertainties associated with it. Instead, we report the slope of the linear regression line through the data, with the root mean square of the error denoted as $\chi$. Figure $7 \mathrm{c}$ shows $\kappa$ and $\chi$ as functions of $S_{t h}$. The error tends to show a minimum in the range $0.5<S_{t h}<0.9$ with values $\chi \approx 0.2$. Note that this range of shape factor values are generally where we see the sharpest gradients in the $S$-profiles plotted in Fig. 7a and also indicated by the superimposed contours in Fig. 6 a.

The minimum error is found at $S_{t h}=0.85$ where the slope is $\kappa=1.89$. From the velocity field, we measure a front propagation factor in the transverse direction as $\kappa_{P I V}=1.96 \pm 0.4$. It should be noted that the speed estimated from the PIV is from the $0.5 U_{c}$ contour, which is itself a surrogate, albeit a commonly used one (Waitukaitis and Jaeger 2012; Peters and Jaeger 2014; Han et al 2016; Peters et al 2016; Majumdar et al 2017; Han et al 2018, 2019b; Baumgarten and Kamrin 2019; Rømcke et al 2021). Thus, our method shows that the propagation of the texture transition and the propagation of the jamming front are comparable.

\subsection{Comparing texture and PIV data for the full field}

Similarities between the texture measurements and the PIV data have been noted in the previous sections. Here, we address the full field of view explicitly. Most notably, the evolution of the region exhibiting surface features (that is $S<0.85$ in Fig. 6a) and the region traversing with the cylinder (that is $u / U_{c}>0.5$ in Fig. $2 \mathrm{e}-\mathrm{h}$ ). In addition, it is observed that the surface features (Fig. 6b) and the eigenvectors of the strain tensor $\left(\mathbf{n}_{1}\right.$ and $\mathbf{n}_{2}$ in Fig. $\left.2 \mathrm{i}-1\right)$ are approximately oriented in the same directions. This section aims to quantify these observations.

The combined evolution of the shape factor and velocity are plotted in Fig. 8. As indicated in Figs. 2 and 6a, the overall trend of the data is to transition from a state of low velocity $\left(u / U_{c}<0.5\right)$ and high shape factor $(S>0.85)$ to a state of high velocity $\left(u / U_{c}>0.5\right)$ and low shape factor $(S<0.85)$. In other words, the suspension transitions from a quiescent suspension with no notable surface features, to moving with the cylinder while exhibiting observable surface features. Importantly, Fig. 8 shows that the transition between these two states occurs at the same time in the experiment.

In addition, we seek to rigorously confirm that the surface features and eigenvectors are oriented in the same direction. This analysis is only relevant where the suspension has deformed sufficiently, and the surface exhibit clear surface features. As such, the data will be separated into regions where the analysis is conducted separately.

As a first step toward identifying the relevant region, Fig. 9a shows the histogram of $S$ and $u / U_{c}$ similar to Fig. 8 for the full time series and all experimental runs. By using the threshold value $S_{t h}=0.85$ for the shape factor (see Sect. 4.2) and the definition of the jamming front $\left(u / U_{c}=0.5\right)$, we divide the data into quadrants. Here, $Q 1$ represents a slow moving texture free state, $Q 2$ represents the transition, while $Q 3$ represents the suspension moving with the cylinder exhibiting measurable surface features. It is worth noting that $Q 1, Q 2$ and $Q 3$ contain roughly 35,15 and $50 \%$ of the data, respectively. The unlabeled quadrant in Fig. 9a contains less than $1 \%$ of the data, and its contribution is negligible. An example of where these regions are located in the flow is presented in Fig. 9b. This figure compares the velocity field in Fig. $2 f$ with the result of the shape factor $S$ acquired from Fig. 2n.

A representation of the texture orientation and the strain eigenvectors are presented in Fig. 10a. The orientations are arbitrary, and the figure is only meant to be illustrative. The vectors are normalized to unit vectors. $\mathbf{n}_{P}$ is defined in Sect. 3.1 and represents the orientation of the texture. As a

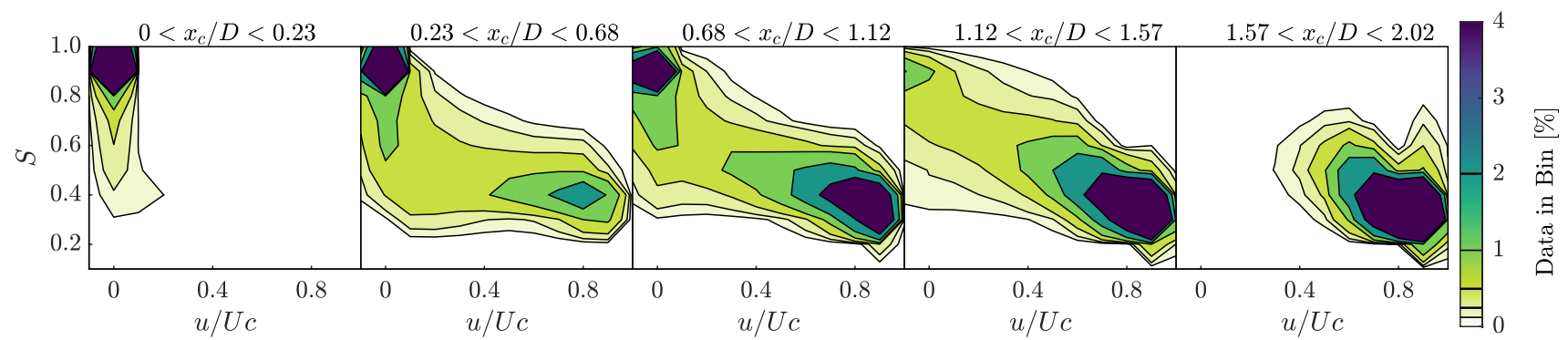

Fig. 8 Evolution of the velocity, $u$, from the PIV experiment and shape factor, $S$, from the texture experiment. The figures are 2D histograms indicating where the data points tend to cluster for different stages in the experiment 


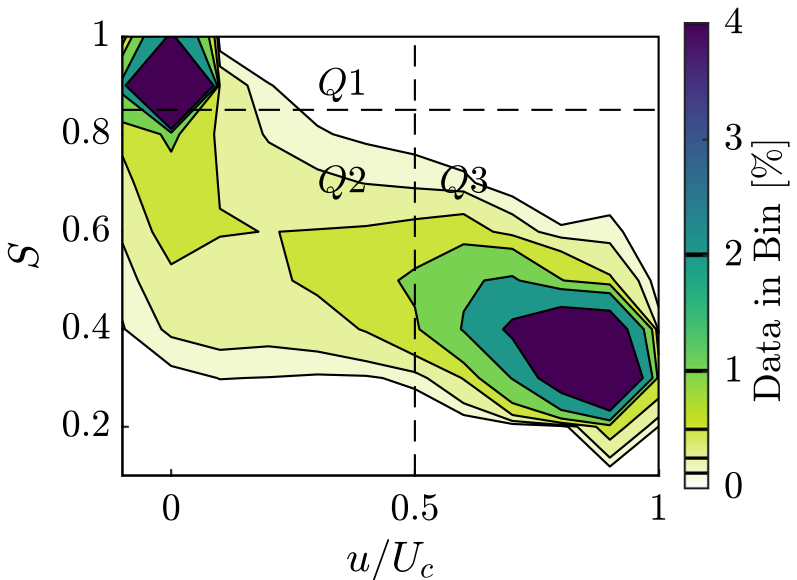

(a)

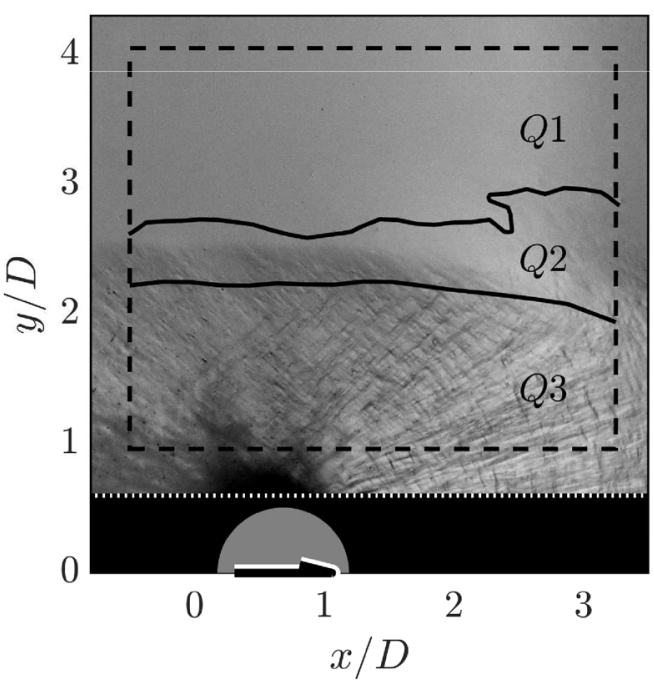

(b)

Fig. 9 a Histogram of $S$ and $u / U_{c}$ similar to Fig. 8 for the whole time series for all experimental runs. The dashed lines separate the data into quadrants $Q 1, Q 2$ and $Q 3$. b Example of how the quadrants represent different regions of the system. This particular snapshot compares the velocity field and texture from Fig. $2 \mathrm{f}$ to $\mathrm{n}$

basis for comparing texture and eigenvector orientation, we will use the dot products $\mathbf{n}_{1} \cdot \mathbf{n}_{P}$ and $\mathbf{n}_{2} \cdot \mathbf{n}_{P}$. Since all three vectors are unit vectors, these dot products represent the cosine of the angle separating them.

Figure $10 \mathrm{~b}$ shows probability density functions (PDF) of $\cos ^{-1}\left(\left|\mathbf{n}_{i} \cdot \mathbf{n}_{P}\right|\right)$ for the regions $Q 1, Q 2$ and $Q 3$, respectively. The absolute value of the dot product is used here, thus representing the angle separating $\mathbf{n}_{P}$ and the span of the eigenvectors $\mathbf{n}_{i}$ with a positive value. As a result, $\cos ^{-1}\left(\left|\mathbf{n}_{i} \cdot \mathbf{n}_{P}\right|\right)=0$ indicates $\mathbf{n}_{P} \| \mathbf{n}_{i}$, while $\cos ^{-1}\left(\left|\mathbf{n}_{i} \cdot \mathbf{n}_{P}\right|\right)=\pi / 2$ indicates $\mathbf{n}_{P} \perp \mathbf{n}_{i}$. As seen

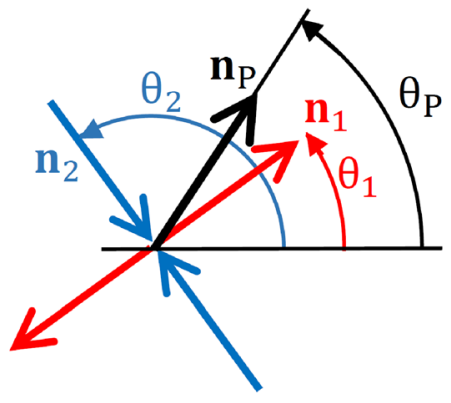

(a)

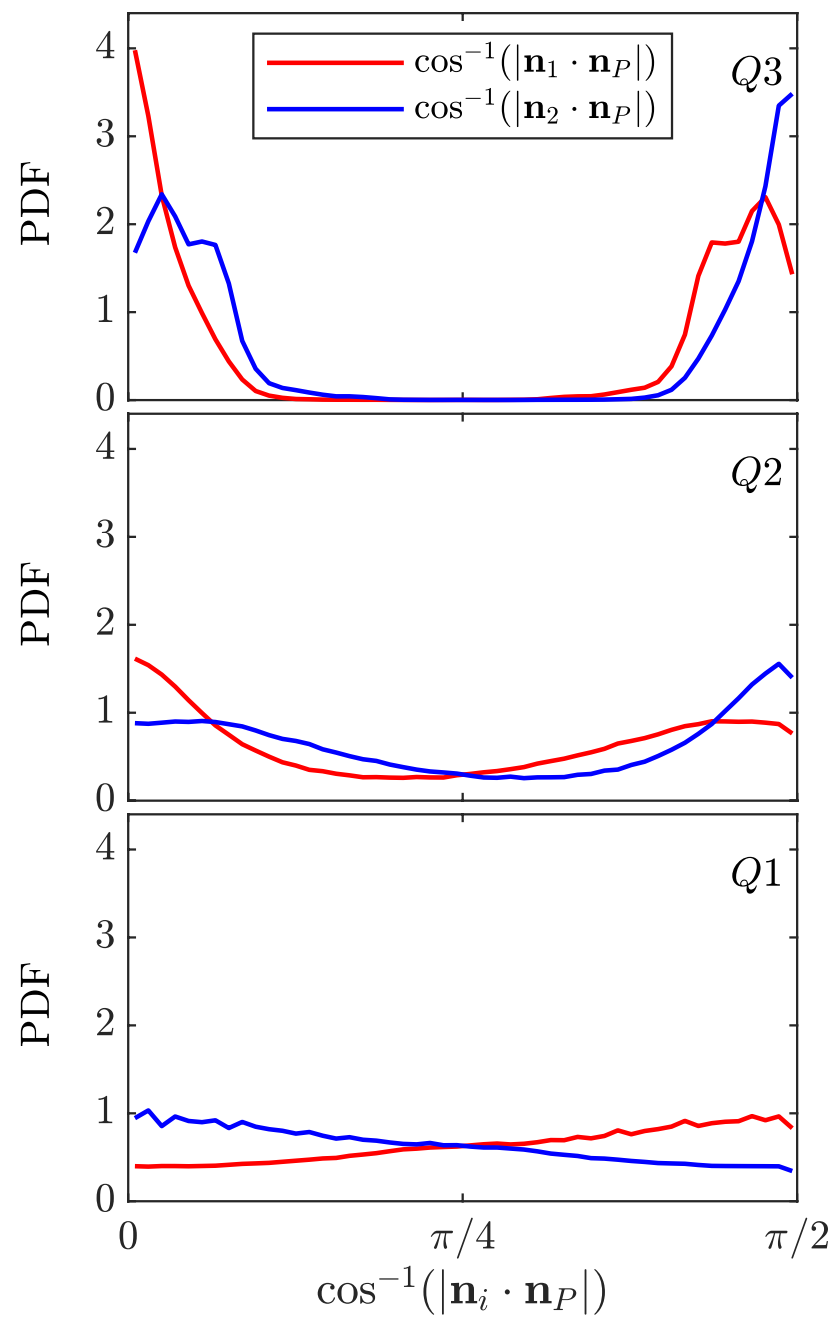

(b)

Fig. 10 a Schematic representation of texture orientation, $\mathbf{n}_{P}$, and strain eigenvectors where $\mathbf{n}_{1}$ represents stretch, and $\mathbf{n}_{2}$ represents compression. b PDFs of the angle separating $\mathbf{n}_{P}$ and $\mathbf{n}_{i}$ for the regions $Q 1, Q 2$ and $Q 3$, respectively 
in Fig. 10b, the texture vectors are somewhat biased toward $\mathbf{n}_{2}$ in the $Q 1$ region. In the transition region, $Q 2$, the texture $\mathbf{n}_{P}$ starts to favor the $\|$ and $\perp$ directions relative to the strain. For $Q 3$, the PDFs are $\approx 0$ in the region $\pi / 8<\cos ^{-1}\left(\left|\mathbf{n}_{i} \cdot \mathbf{n}_{P}\right|\right)<3 \pi / 8$ with strong peaks at 0 and $\pi / 2$. In other words, the PDFs from region $Q 3$ clearly show that the texture observed at the free surface has a strong connection with the orientation of the eigenvectors of the strain tensor.

\section{Conclusion}

Measurements of how surface texture evolves on a dense suspension of cornstarch and water with a freely propagating shear jamming front have been presented. The surface texture is captured by high-speed images of the free surface looking into a direct reflection. PIV measurements are used as a reference. From images of the surface texture, a 2D fast Fourier transform of local interrogation windows is used as a basis for analysing the surface structure. By taking a sector average of the power spectra, we are able to identify whether surface features are observed and the direction that they are oriented.

The PIV and texture measurements are two separate experiments, however, we show that the region of the suspension that shows clear surface features overlaps with the jammed region. In addition, we show that in the jammed, textured region of the flow, the eigenvectors of the strain tensor, and the observed surface features are oriented in the same direction. Hence, our analysis reveals that pictures of the free surface contain quantifiable information not previously directly accessed.

Dilation (Brown and Jaeger 2012; Jerome et al 2016; Majumdar et al 2017; Maharjan et al 2021) as well as surface corrugations (Loimer et al 2002; Timberlake and Morris 2005) have been observed at the free surface of dense suspensions before. However, few studies have investigated the surface texture at a freely propagating shear jamming front (Allen et al 2018). The results presented in the current work, particularly the relation between the eigenvectors of the strain tensor and the orientation of the surface features, will provide insight for future model development and understanding of dense suspensions as well as a valuable measurement tool for future investigations.

Acknowledgements RJH is funded by the Research Council of Norway through Project No. 288046. IRP acknowledges financial support from the Royal Society (Grant No. RG160089). Data supporting this study are openly available from the University of Southampton repository (https://doi.org/10.5258/SOTON/D1973).
Funding Open access funding provided by NTNU Norwegian University of Science and Technology (incl St. Olavs Hospital - Trondheim University Hospital).

Open Access This article is licensed under a Creative Commons Attribution 4.0 International License, which permits use, sharing, adaptation, distribution and reproduction in any medium or format, as long as you give appropriate credit to the original author(s) and the source, provide a link to the Creative Commons licence, and indicate if changes were made. The images or other third party material in this article are included in the article's Creative Commons licence, unless indicated otherwise in a credit line to the material. If material is not included in the article's Creative Commons licence and your intended use is not permitted by statutory regulation or exceeds the permitted use, you will need to obtain permission directly from the copyright holder. To view a copy of this licence, visit http://creativecommons.org/licenses/by/4.0/.

\section{References}

Albrecht M, Christiansen S, Michler J, Dorsch W, Strunk HP, Hansson PO, Bauser E (1995) Surface ripples, crosshatch pattern, and dislocation formation: cooperating mechanisms in lattice mismatch relaxation. Appl Phys Lett 67(9):1232-1234. https://doi.org/10. 1063/1.115017

Allen B, Sokol B, Mukhopadhyay S, Maharjan R, Brown E (2018) System-spanning dynamically jammed region in response to impact of cornstarch and water suspensions. Phys Rev E 97(052):603. https://doi.org/10.1103/PhysRevE.97.052603

Baumgarten AS, Kamrin K (2019) A general constitutive model for dense, fine-particle suspensions validated in many geometries. Proc National Academy Sci 116(42):20,828-20,836. https://doi. org/10.1073/pnas.1908065116

Bischoff White EE, Chellamuthu M, Rothstein JP (2010) Extensional rheology of a shear-thickening cornstarch and water suspension. Rheol Acta 49(2):119-129

Brown E, Jaeger HM (2012) The role of dilation and confining stresses in shear thickening of dense suspensions. J Rheol 56(4):875-923. https://doi.org/10.1122/1.4709423

Brown E, Jaeger HM (2014) Shear thickening in concentrated suspensions: phenomenology, mechanisms and relations to jamming. Reports Prog Phys 77(4):046,602

Cates ME, Wyart M (2014) Granulation and bistability in non-brownian suspensions. Rheol Acta 53(10):755-764

Cates ME, Haw MD, Holmes CB (2005) Dilatancy, jamming, and the physics of granulation. J Phys: Condens Matter 17(24):S2517S2531. https://doi.org/10.1088/0953-8984/17/24/010

Chang KH, Gilbala R, Srolovitz DJ, Bhattacharya PK, Mansfield JF (1990) Crosshatched surface morphology in strained iii-v semiconductor films. J Appl Phys 67(9):4093-4098. https://doi.org/ 10.1063/1.344968

Denn MM, Morris JF, Bonn D (2018) Shear thickening in concentrated suspensions of smooth spheres in Newtonian suspending fluids. Soft Matter 14:170-184. https://doi.org/10.1039/C7SM00761B

Fernandez N, Mani R, Rinaldi D, Kadau D, Mosquet M, LomboisBurger H, Cayer-Barrioz J, Herrmann HJ, Spencer ND, Isa L (2013) Microscopic mechanism for shear thickening of nonBrownian suspensions. Phys Rev Lett 111(108):301. https://doi. org/10.1103/PhysRevLett.111.108301

Gadala-Maria F, Acrivos A (1980) Shear-induced structure in a concentrated suspension of solid spheres. J Rheol 24(6):799-814. https://doi.org/10.1122/1.549584

Guazzelli E, Pouliquen O (2018) Rheology of dense granular suspensions. J Fluid Mech 852:P1. https://doi.org/10.1017/jfm.2018.548 
Guy BM, Hermes M, Poon WCK (2015) Towards a unified description of the rheology of hard-particle suspensions. Phys Rev Lett 115(088):304. https://doi.org/10.1103/PhysRevLett.115.088304

Han E, Peters IR, Jaeger HM (2016) High-speed ultrasound imaging in dense suspensions reveals impact-activated solidification due to dynamic shear jamming. Nature Commun 7(1), https://doi.org/ 10.1038/ncomms 12243

Han E, Wyart M, Peters IR, Jaeger HM (2018) Shear fronts in shearthickening suspensions. Phys Rev Fluids 3(073):301. https://doi. org/10.1103/PhysRevFluids.3.073301

Han E, James NM, Jaeger HM (2019a) Stress controlled rheology of dense suspensions using transient flows. Phys Rev Lett 123(248):002. https://doi.org/10.1103/PhysRevLett.123.248002

Han E, Zhao L, Van Ha N, Hsieh ST, Szyld DB, Jaeger HM (2019b) Dynamic jamming of dense suspensions under tilted impact. Phys Rev Fluids 4(063):304. https://doi.org/10.1103/PhysRevFluids.4. 063304

James NM, Han E, Cruz RAL, Jureller J, Jaeger HM (2018) Interparticle hydrogen bonding can elicit shear jamming in dense suspensions. Nature Mater 17. https://doi.org/10.1038/ s41563-018-0175-5

Jerome JJS, Vandenberghe N, Forterre Y (2016) Unifying impacts in granular matter from quicksand to cornstarch. Phys Rev Lett 117(098):003. https://doi.org/10.1103/PhysRevLett.117.098003

Krieger IM (1972) Rheology of monodisperse latices. Adv Coll Interface Sci 3(2):111-136. https://doi.org/10.1016/0001-8686(72) 80001-0

Kumar AA, Medhi BJ, Singh A (2016) Experimental investigation of interface deformation in free surface flow of concentrated suspensions. Phys Fluids 28(11):113,302. https://doi.org/10.1063/1. 4967739

Lin NYC, Guy BM, Hermes M, Ness C, Sun J, Poon WCK, Cohen I (2015) Hydrodynamic and contact contributions to continuous shear thickening in colloidal suspensions. Phys Rev Lett 115(228):304. https://doi.org/10.1103/PhysRevLett.115.228304

Loimer T, Nir A, Semiat R (2002) Shear-induced corrugation of free interfaces in concentrated suspensions. J Non-Newtonian Fluid Mech 102(2):115-134, https://doi.org/10.1016/S03770257(01)00173-2, https://www.sciencedirect.com/science/artic le/pii/S0377025701001732, a Collection of Papers Dedicated to Professor ANDREAS ACRIVOS on the Occasion of his Retirement from the Benjamin Levich Institute for Physiochemical Hydrodynamics and the City College of the CUNY

Madraki Y, Hormozi S, Ovarlez G, Guazzelli E, Pouliquen O (2017) Enhancing shear thickening. Phys Rev Fluids 2(033):301. https:// doi.org/10.1103/PhysRevFluids.2.033301

Maharjan R, O’Reilly E, Postiglione T, Klimenko N, Brown E (2021) Relation between dilation and stress fluctuations in discontinuous shear thickening suspensions. Phys Rev E 103(012):603. https:// doi.org/10.1103/PhysRevE.103.012603

Majumdar S, Peters IR, Han E, Jaeger HM (2017) Dynamic shear jamming in dense granular suspensions under extension. Phys Rev E 95(012):603. https://doi.org/10.1103/PhysRevE.95.012603

Mari R, Seto R, Morris JF, Denn MM (2014) Shear thickening, frictionless and frictional rheologies in non-Brownian suspensions. $\mathrm{J}$ Rheol 58(6):1693-1724. https://doi.org/10.1122/1.4890747
Mukhopadhyay S, Allen B, Brown E (2018) Testing constitutive relations by running and walking on cornstarch and water suspensions. Phys Rev E 97(052):604. https://doi.org/10.1103/PhysR evE.97.052604

Nemat-Nasser S (2004) Plasticity : a treatise on finite deformation of heterogeneous inelastic materials. Cambridge monographs on mechanics. Cambridge University Press, Cambridge

Peters IR, Jaeger HM (2014) Quasi-2d dynamic jamming in cornstarch suspensions: visualization and force measurements. Soft Matter 10:6564-6570. https://doi.org/10.1039/C4SM00864B

Peters IR, Majumdar S, Jaeger HM (2016) Direct observation of dynamic shear jamming in dense suspensions. Nature 532(7598):214-217, http://search.proquest.com/docview/17815 36995/

Rømcke O, Peters IR, Hearst RJ (2021) Getting jammed in all directions: dynamic shear jamming around a cylinder towed through a dense suspension. Phys Rev Fluids 6(063):301. https://doi.org/ 10.1103/PhysRevFluids.6.063301

Singh A, Nir A, Semiat R (2006) Free-surface flow of concentrated suspensions. Int J Multiphase Flow 32(7):775-790

Singh A, Mari R, Denn MM, Morris JF (2018) A constitutive model for simple shear of dense frictional suspensions. J Rheol 62(2):457468. https://doi.org/10.1122/1.4999237

Sivadasan V, Lorenz E, Hoekstra AG, Bonn D (2019) Shear thickening of dense suspensions: The role of friction. Physics of Fluids 31(10):103,103. https://doi.org/10.1063/1.5121536

Smith M, Besseling R, Cates M, Bertola V (2010) Dilatancy in the flow and fracture of stretched colloidal suspensions. Nature Commun 114. https://doi.org/10.1038/ncomms1119

Stickel JJ, Powell RL (2005) Fluid mechanics and rheology of dense suspensions. Annu Rev Fluid Mech 37(1):129-149. https://doi. org/10.1146/annurev.fluid.36.050802.122132

Tapia F, Pouliquen O, Guazzelli E (2019) Influence of surface roughness on the rheology of immersed and dry frictional spheres. Phys Rev Fluids 4(104):302. https://doi.org/10.1103/PhysRevFluids.4. 104302

Timberlake BD, Morris JF (2005) Particle migration and free-surface topography in inclined plane flow of a suspension. J Fluid Mech 538:309-341. https://doi.org/10.1017/S0022112005005471

Waitukaitis SR, Jaeger HM (2012) Impact-activated solidification of dense suspensions via dynamic jamming fronts. Nature 487(7406):205. https://doi.org/10.1038/nature11187

Wyart M, Cates ME (2014) Discontinuous shear thickening without inertia in dense non-Brownian suspensions. Phys Rev Lett 112(098):302. https://doi.org/10.1103/PhysRevLett.112.098302

Zhang X (1996) An algorithm for calculating water surface elevations from surface gradient image data. Exp Fluids 21(1):43-48

Publisher's Note Springer Nature remains neutral with regard to jurisdictional claims in published maps and institutional affiliations. 